\title{
Analysis of Mechanical Properties of Composite Materials with Nano Particles by using Feathers
}

\author{
G. Bhargav ${ }^{1}$, N. Srikanth ${ }^{2}$, K. Vinay Raj ${ }^{3}$, S. Sai Teja ${ }^{4}$, M. Bhojendra Naik ${ }^{5}$ \\ 1,2,3,4 Department of Mechanical Engineering, St. Martin's Engineering College, Hyderabad, \\ 5. Assistant Professor, Department of Mechanical Engineering, St. Martin's Engineering College, Hyderabad, India.
}

\begin{abstract}
A material may be a material made up of two or more constituent materials with the opposite significantly different physical or chemical properties that, once they are combine to supply a material with characteristics different from the individual components. The individual component materials remain separate and the distinct properties within the final structure. The new material could also be preferred for several reasons: common examples include materials which are stronger, lighter, or less costly in comparison to traditional materials. In this study composite materials are prepared using chicken feathers in the matrices of using the Nano-metallic particles of aluminium oxide \& resin\& hardener also used in preparing the sheet. The mechanical properties are tested and compared with the other composite materials
\end{abstract}

Key words: Composite material, less expensive, traditional materials, stronger, lighter in weight, Nano metallic particles of aluminium oxide, Resin \& hardener

\section{INTRODUCTION:}

A material often defined as a mixture of two or more materials that leads to better properties than those of the individual components used alone. In contrast to Nanometallic particles of aluminium oxide (al2o3) and the chicken feather as another composite material each material retains its separate chemical, physical, and mechanical properties. The two constituents are reinforcement and a matrix. The advantages of the composite materials have the higher strength and stiffness to the materials, combined with rarity, as compared with bulk materials, allowing a weight reduction within the finished part. The resin and hardener phase provides the clear surface finish and it has good mechanical properties. In most cases the resin and hardener are stronger, stiffer and light in weight the matrix arrangement. The resin hardens within 24 hours. With the composite materials added to it the Feather composites have different dimensions in the sizes and chopped one also added to the sheet to mix with the Nano metallic particles of the aluminium to the sheet and it gives the good mechanical properties of the composite materials. The sheets are in square and round any other regular or irregular geometry is used. The feathers are arranged in the different positions in continuous and discontinuous method arrangements the sheets with filled epoxy resin and hardener, Nano-metallic particles of Aluminium is used and formed a matrix sheet in the square \&round shape. In equal dimension and it as a good strength and mechanical properties to the sheet. Feathers produce high-strength composites because of their smaller in size and it has a keratin protein which gives abundantly strength to the matrix sheet in the arrangement of the feather. As a general rule, the smaller in size of the feather, the higher its strength and also the feather we have used directly with the whole feather and chopped feather into small pieces to give the good strength and mixed with the Nano particles of aluminium oxide. Continuous phase is that the matrix, which may be a polymer, metal, or ceramic. Polymers have less strength and stiffness, metals have medium strength and stiffness but high ductility, The matrix (continuous phase) performs several critical functions, including maintaining the feathers in the proper orientation and spacing and protecting them from abrasion and the environment. In polymer and metal matrix composites that form a strong bond between the feather and the matrix, the matrix transmits loads from the matrix to the feather through shear loading at the interface. In ceramic matrix composites, the target is usually to extend the toughness instead of the strength and stiffness; therefore, a coffee interfacial strength bond is desirable. The type and quantity of the reinforcement determine the final properties. Figure 1.2 shows that the highest strength and modulus are obtained with continuous-fiber composites. There is a practical limit of about 70 volume percent reinforcement which will be added to make a composite. At higher percentages, there is too little matrix to support the feathers effectively. Theoretical strength of discontinuous-feathers composites can approach

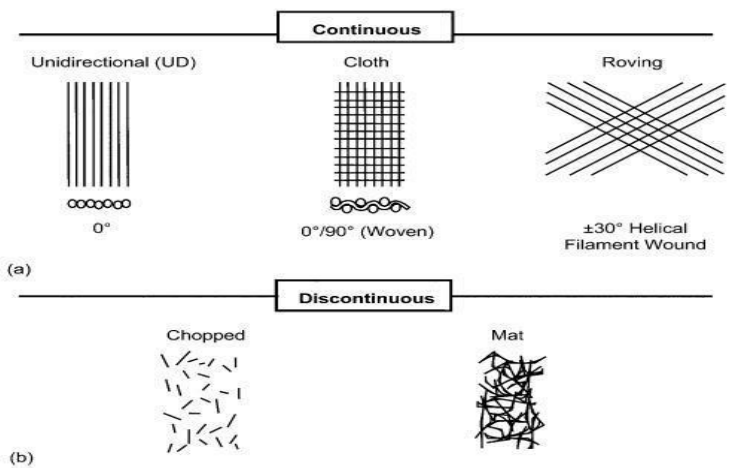

Fig.1. Typical reinforcement types

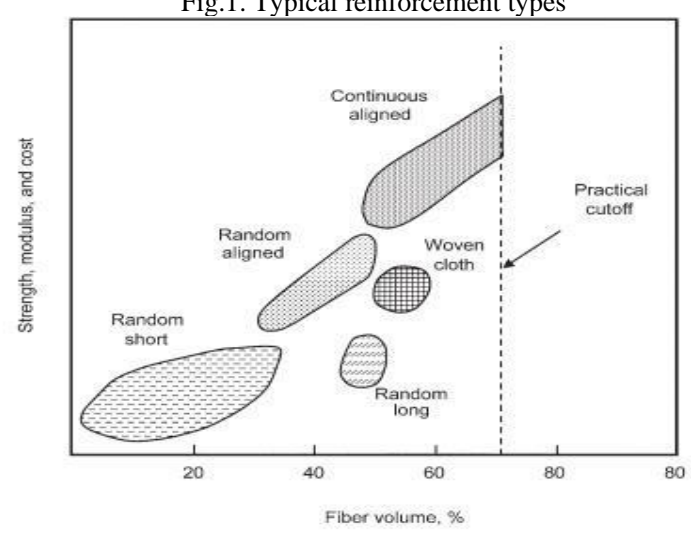

Fig.2. Influence of reinforcement type and quantity on composite performance 
That of continuous-feather composites if their aspect ratios are great enough and they are aligned, but it is difficult in practice to maintain good alignment with discontinuous feathers. Discontinuous-feather composites are normally somewhat random in alignment, which dramatically reduces their strength and modulus. However, discontinuousfeathers composites are generally much less than continuous-feather composites. Therefore, continuousfeather composites are used where higher strength and stiffness are required (but at a higher cost), and discontinuous-feather composites are used where cost is the main driver and strength and stiffness are less important. Both the reinforcement type and therefore the matrix affect processing. The major process for the polymer matrices composites are shown in Fig. 1.3. Two types of polymer matrices are shown: thermo sets and thermoplastics. A thermo set starts as a low-viscosity resin that reacts and cures during processing, forming an intractable solid. A thermoplastic may be a high-viscosity resin that's processed by heating it above its melting temperature. Because a thermo set resin sets up and cures during processing, it can't be reprocessed by reheating. By comparison, a thermoplastic are often reheated above its melting temperature for extra processing. There are processes for both classes of resins that are more amenable to discontinuous feathers \& continuous feathers

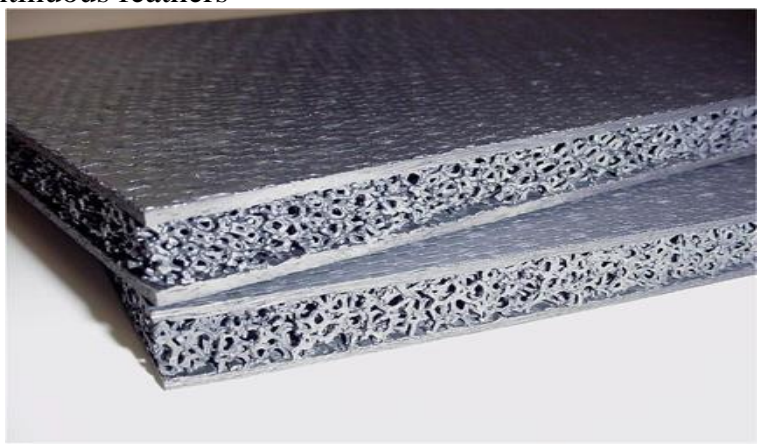

Fig.3. Ceramic matrix composites

Ceramic matrix - Ceramic matrix composites (CMCs) are a Sub-group of composite materials. They contains ceramic fibers embedded during a ceramic matrix, thus forming a ceramic fiber reinforced ceramic (CFRC) material. The matrix and fibers can contains any ceramic material. CMC materials are of low brittleness, less fracture toughness, and less shock resistance faced by the traditional technical ceramics. Metal matrix: Metal matrix composites (MMCs) are Composite materials that contain a minimum of two constituent parts ??? a metal and another material or a special metal. The metal matrix is reinforced with the opposite material to enhance strength and wear. Where three or more constituent parts are present, it's called a hybrid composite. In structural applications, the matrix is typically composed of a lighter metal like magnesium, titanium, or aluminium. In heat applications, cobalt and cobalt-nickel alloy matrices are common. Typical MMC's manufacturing is essentially divided into three types: solid, liquid, and vapour. Continuous carbon, carbide, or ceramic fibers are a number of the materials which will be embedded during a metallic matrix material. MMCs are fire resistant, operate during a wide selection of temperatures, don't absorb moisture, and possess better electrical and thermal conductivity. They have also found applications to be resistant to radiation damage, and to not suffer from outgassing. Most metals and alloys observe matrices for composite applications.

\section{METHODOLOGY:}

To make our comparative analysis we took three different proportions of the materials with the feathers random arrangement three different epoxy resin \& hardener for different arrangements of the feathers.

The three different proportions taken for the analysis of mechanical properties of the composite material are:

a. Chicken feathers

b. Nano metallic particles of Aluminium oxide

c. Resin and hardener

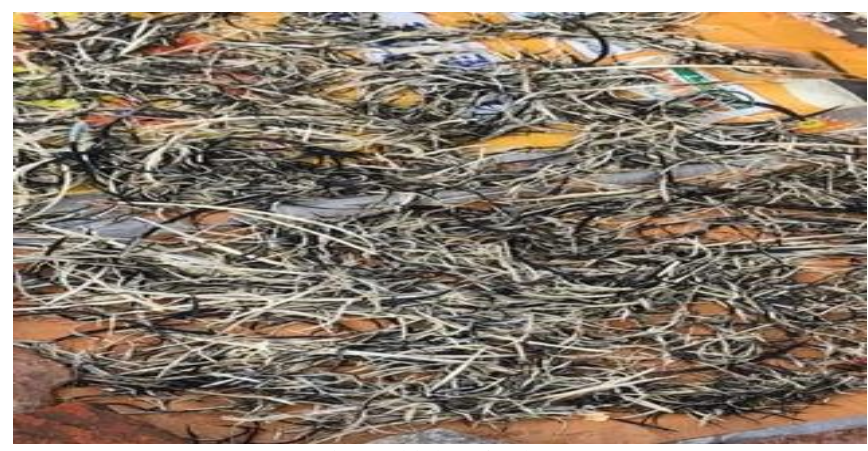

Fig.4. chicken feather

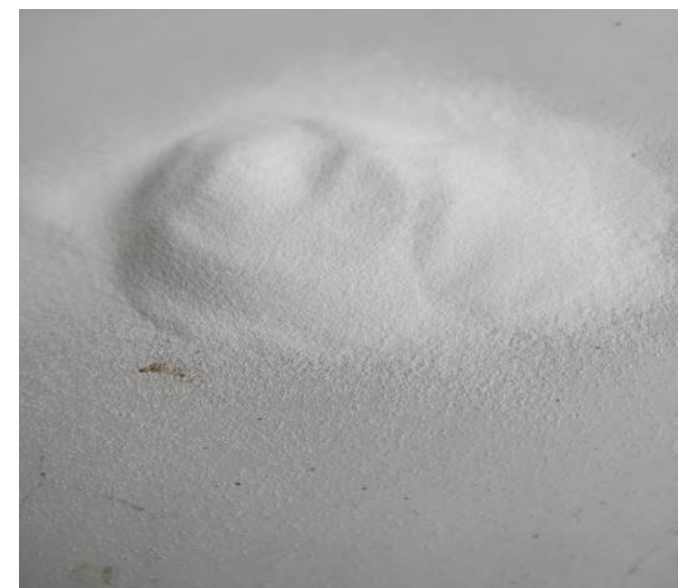

Fig.5. Nano metallic particles of the aluminium oxide

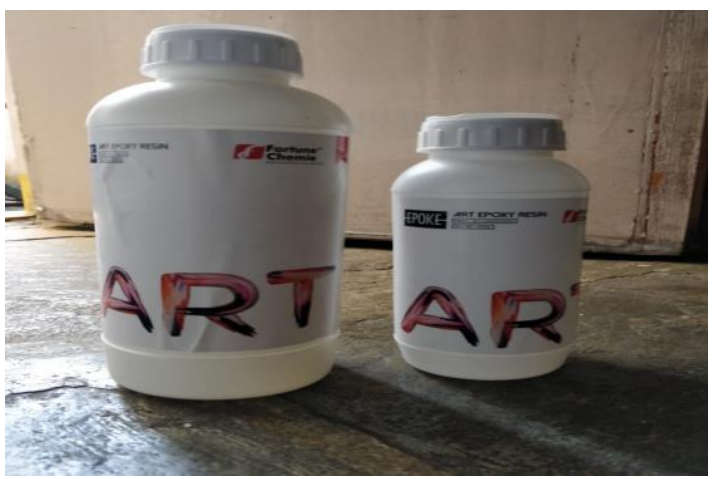

Fig.6. resin and hardener 


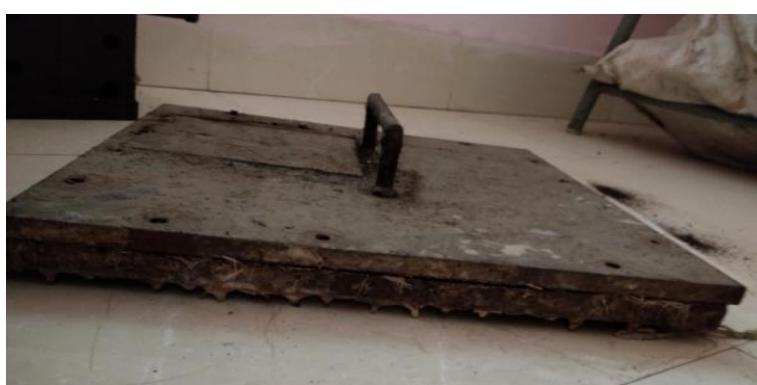

Fig.7. the casting die

The different proportions' of the materials taken for the different arrangement of material for the analysis of mechanical properties of the composite material is as given the following table.

Table no 1: Different proportions of the mixture

\begin{tabular}{|c|c|c|c|}
\hline S. no & \multicolumn{1}{|l|}{ Resin } & Hardener & Al2o3 \\
\hline 1 & $400 \mathrm{ml}$ & $130 \mathrm{ml}$ & $100 \mathrm{grams}$ \\
\hline 2 & $450 \mathrm{ml}$ & $160 \mathrm{ml}$ & 125 grams \\
\hline 3 & $500 \mathrm{ml}$ & $180 \mathrm{ml}$ & 150 grams \\
\hline
\end{tabular}

The orientation arrangements taken done for the sheet producing

a. Random (Mechanical mixing)

As we said that we are going to make composite material mixture of using three different material. The following epoxy resin was used for different arrangement and different fibers.

Chicken Feathers: Chicken feathers was taken from the local source from nearby location. The feathers was collected and it was cleaned thoroughly with shampoo and dried in the sun shade for a week for bad odours from the feathers to leave off. The feathers shouldn't be touched directly through the hand. Surgical gloves should be wore to the hands till the project is done as the resin and hardener is chemically not good to the skin. So, the gloves are very mandatory.

We need to have mould. The mould has cope and drag. The upper mould has the dimensions of the part material sheet (384mmx384mmx12mm) and the lower mould has the dimension of $(384 \mathrm{~mm} \times 384 \mathrm{~mm} \times 16 \mathrm{~mm})$ Then the resulting sheet will be of $(300 * 300 * 3)$.

\section{PROCEDURE OF TESTING:}

a) Tensile test: First of all the specimen is cut in required shape. For this testing we have taken the specimen in the dumbbell shape as shown in the figure below. The specimens were cut through electronic machine. Then the specimen is taken to the ultimate tensile strength machine. The specimen is hold on either sides of the machine .Then the load is applied on the specimen. The specimen is pulled on the either sides of the ultimate tensile strengthen machine.

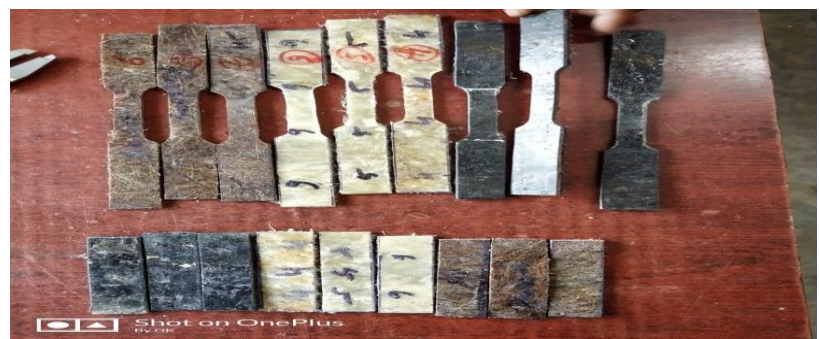

Fig.8. required shapes for cutting

At the certain point, the specimen breaks. The breakpoint of the sheet shows the results of the tensile strength of the chicken feathers in orientation.

Therefore, the reading is taken through the computer which is attached to the machine

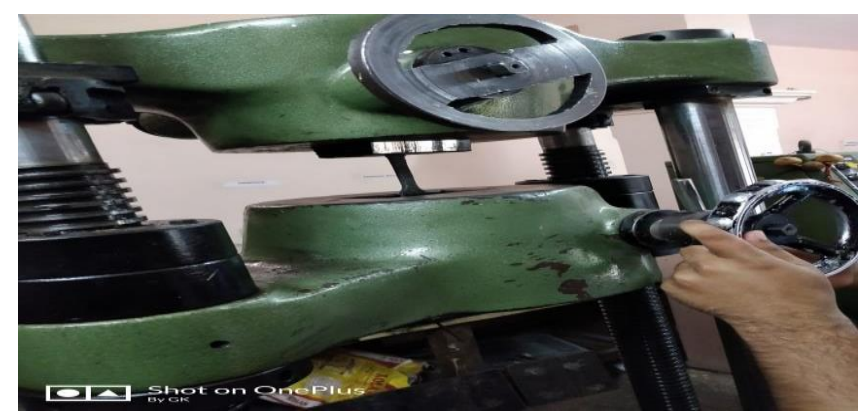

Fig.9 Tensile testing

\section{b. Flexural test:}

The specimen is cut into required shape. The shape taken for flexural test is rectangular shape. The cutting process is done through the electronic machine. Then the specimen is taken to the flexural testing machine. Then the tool is attached to the 2-point machine.

Then give the required power supply to the machine the machine starts working the tool moves towards the specimen and specimen breaks at certain point and reading is taken through computer.

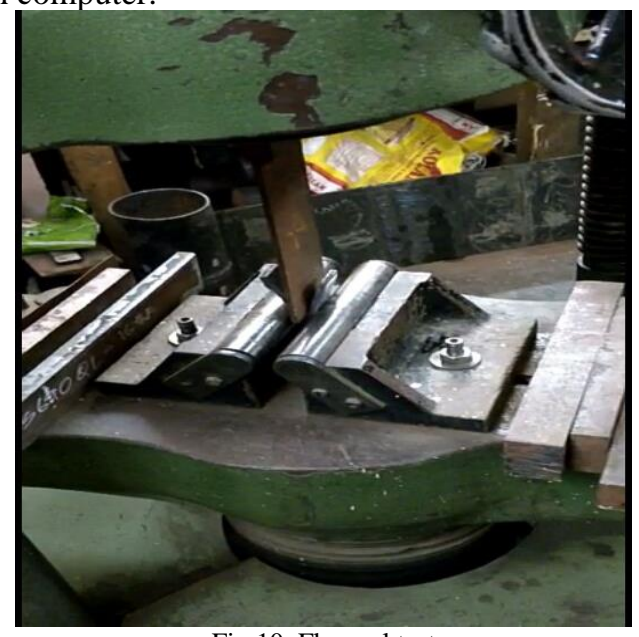

Fig.10. Flexural test

c. Hardness test: Durometer Hardness is used to determine the relative hardness of soft materials, usually plastic or rubber. This test is used to measure the penetration of a specified indentor into the material under the specific 
conditions of force and time. The hardness value is often used to identify the harness of the elastomer and its quality control.

The specimen is first placed on a hard flat surface. The indentor of instrument is penetrates on the sheet pressed into the specimen making sure that it is parallel to the surface. The hardness can be noted in less than a second and the person in contact with machine can be notified.

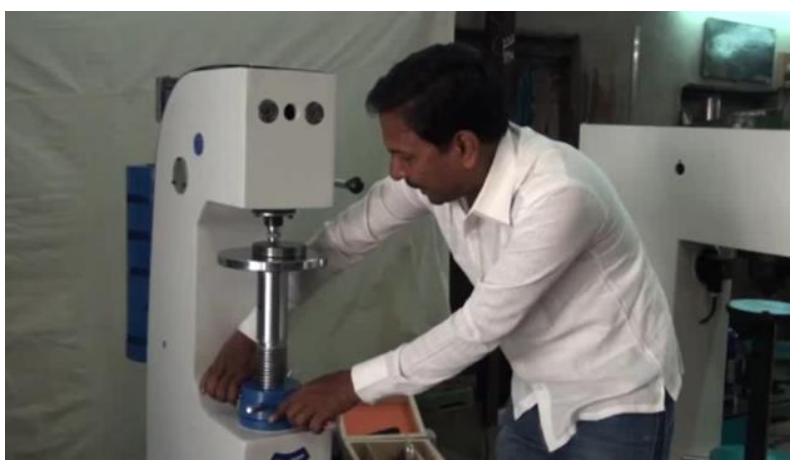

Fig.11. hardness testing machine

\section{RESULT AND DISCUSSION:}

\section{Random arrangement:}

The graph of the Ultimate tensile strength, Flexural strength and Hardness test of the Chicken feathers materials with the mixture of nano metallic particles of the aluminium oxide and the resin and hardener in the mixture i.e., random arrangement.

In the mechanical mixing and arranging the order of random distribution of feathers .Here the results are getting the different according to feathers we are using in the way of arranging.

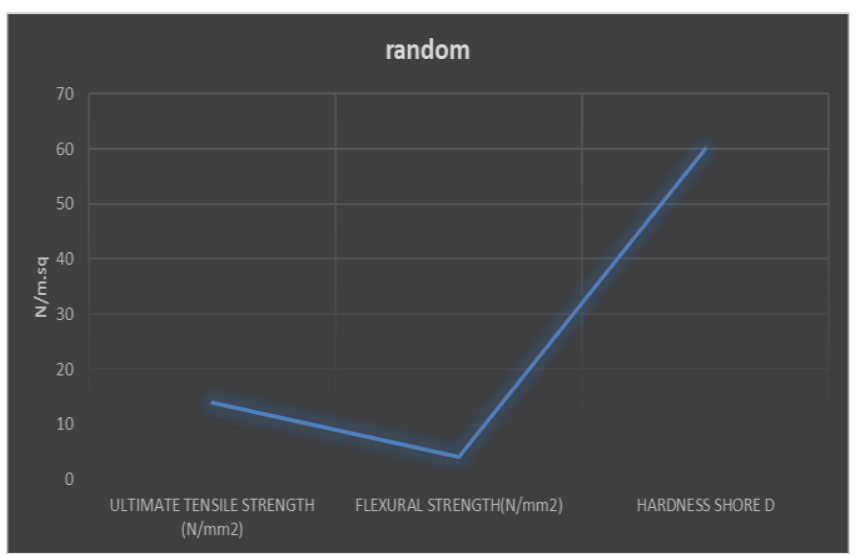

Fig.12. Testing of Random arrangement of feathers

\section{CONCLUSION:}

Composites are most promising materials for components of current and future engineering structures, with a significant demand at present in aircraft and aerospace industries. Modal analysis is that the study of the natural characteristics of structures. Understanding both the natural frequency and mode shape helps to style any structural system for noise and vibration applications. In this project the analysis of the cantilever beam and other material holding for the composite material as well as steel material are carried out.
Here we prepared composite materials using the chicken feathers, Resin \& hardener, Nano-metallic particles of aluminium oxide.

1. It is seen that the different resins have different influence on the hardness and tensile strength of the composite materials.

2. And also the arrangement of feathers in the sheet also effects the hardness of the composite materials.

3. Next it effects the different areas of sheet of the mixture of the material Nano metallic particles of the aluminium oxide (al203) which we are using for the composite materials to give the strength to the sheet. 4. And finally we are getting better results in the hardness for the feathers in the sheet.

\section{ACKNOWLEDGMENT}

I wish to thank Assistant Prof. M.BHOJENDRA NAIK for his guidance throughout the study. I also extend my gratitude to my friends in helping the project work and research works.

\section{REFERENCES:}

[1] Chnepf, M.J.; Mayer, M.; Kuttner, C.; Tebbe, M.; Wolf, D.; Dulle, M.; Altantzis, T.; Formanek, P.; Förster, S.; Bals, S.; König, T.A.F.; Fery, A. (2017). "Nanorattles with tailored electric field enhancement". Nanoscale. 9 (27): 9376-9385.

[2] Layton May Epoxy Resins: Chemistry and Technology Second Edition, 0824776909 p.784 1987 "Epoxies are the resins most commonly employed for electrical and electronic applications. Selection of epoxies is based on their superior adhesion, permeability, purity, and corrosion- and stress-resistance properties."

[3] Jump up to:a b Hakiki, Farizal et al. (2015). Is Epoxy-Based Polymer Suitable for Water Shut-Off Application? SPE-176457MS. SPE/IATMI Asia Pacific Oil \& Gas Conference and Exhibition, 20-22 October, Nusa Dua, Bali, Indonesia. doi: 10.2118/176457-MS

[4] Yin Li, Wei Zhang, An-bo Ming, Zheng-wei Yang, GanTian, "A new way for revealing the damage evolution of impacted CFRP laminate under compression-compression fatigue load based on thermographic images, Composite Structures,2017, Published by Elsevier Ltd. [2] MelisYurddaskal, UgurOzmen, Mehmet Kir, BuketOkutan Baba, "The Effect of Foam Properties on Vibration Response of Curved Sandwich Composite Panels", Composite Structures, 2017, Published by Elsevier Ltd. 